\title{
Characterization of Extremal Unicyclic Graphs Using F-Coindex
}

\author{
Muhammad Imran $\left(D,{ }^{1}\right.$ Yasir Ali, ${ }^{2}$ Zainab Bibi, ${ }^{3}$ and Muhammad Saad Ghafar ${ }^{4}$ \\ ${ }^{1}$ Department of Mathematical Sciences, United Arab Emirates University, P.O. Box 15551, Al Ain, UAE \\ ${ }^{2}$ College of Electrical and Mechanical Engineering, National University of Sciences and Technology, Islamabad, Pakistan \\ ${ }^{3}$ School of Natural Sciences, National University of Sciences and Technology, Islamabad, Pakistan \\ ${ }^{4}$ Department of Mathematics, National University of Modren Languages, Rawalpindi, Pakistan \\ Correspondence should be addressed to Muhammad Imran; imrandhab@gmail.com
}

Received 31 May 2021; Accepted 24 July 2021; Published 26 August 2021

Academic Editor: Muhammad Javaid

Copyright (c) 2021 Muhammad Imran et al. This is an open access article distributed under the Creative Commons Attribution License, which permits unrestricted use, distribution, and reproduction in any medium, provided the original work is properly cited.

\begin{abstract}
The study of forgotten index and coindex for the molecular structures of some special chemical graphs (compounds and drugs) has proven significant in medical and pharmaceutical drug design fields by making reliable statistical conclusion about biological properties of new chemical compounds and drugs. In mathematical chemistry, finding extreme graphs with respect to topological index is an active area of research. The aim of this paper is to characterize the family of unicyclic graphs with extreme (largest and smallest) F-coindex. Moreover, the study also contains different other properties of unicyclic graphs. All these results are based upon an alternative form of $F$-coindex which is established with the help of special property in the graphs.
\end{abstract}

\section{Introduction}

There is a continuous rise in various new diseases every year, the reason being different novel viruses and bacteria. In order to overcome such diseases, introduction of highquality drugs is of prime significance. Importance of topological indices is a well-established fact for nanotechnology in isomer discrimination, pharmaceutical drug design, structure-property relationship, and structure-activity relationship (for details, see [1-3]). In 1972, Gutman and Trinajstic [4] approximated formulas for the total $\pi$-electron energy $(E)$. Among the formulas presented in [4], a pair of topological indices was studied more frequently than any other indices. These pairs of indices are known as Zagreb first and second indices denoted by symbols $M_{1}$ and $M_{2}$, respectively. A detailed study of these indices can be found in [5]. Other than $M_{1}$ and $M_{2}$, another formula depending upon sum of cubes of vertex degrees was also presented in [4]. In order to shed some light on this important index, Furtula and Gutman [6] established some of its basic properties of $F$-index ("forgotten topological index"). Furtula and Gutman [6] further showed that $F$-index can improve physicochemical applicability of $M_{1}$, significantly. Also, by assuming the linear model, they enhanced the predictive ability of these indices. While working on weighted Wiener polynomial of graphs, Doslic [7] initiated work on coindices by introducing Zagreb coindices. Formal definitions of Zagreb coindices and basic properties were reported by Ashrafi et al. [8]. De et al. [9] studied some key features of F-coindex. In [9], the authors compared the correlation of the logarithm of the octanol-water partition coefficient $(P)$ with $F$-coindex, $M_{1}$, and $F$. They came out with result that correlation coefficient between $\log P$ and $F$ coindex is stronger than that of $M_{1}$ and $F$. They concluded that $F$-coindex can predict the $\log P$ values with high accuracy. The study of $F$-index and $F$-coindex is an active area of research nowadays, and we can find a number of results, for example, [10-14]. Recently, Amin et al. [15] studied about minimal trees using F-coindex. The results in [15] are only about first, second, and third minimum trees. Very recently, Akther et al. [16] deduced the results for extremal graphs for F-index among the classes of connected unicyclic and bicyclic graphs. In the present work, we provide an alternative form of F-coindex to study different properties of unicyclic graphs. Moreover, these properties are further used to study extremal graphs for $F$-coindex for the family of unicyclic graphs. 
Throughout the work we assume that $G(V(G), E(G))$ be a graph where $V(G)$ and $E(G)$, respectively, denote vertex and edge sets. Also, $G(V(G), E(G))$ is a connected graph with order $|V(G)|$ and size $|E(G)|$. An adjacent edge is denoted by $e=u v \in E(G)$, whereas $u w \notin E(G)$ is used to denote that $u$ and $w$ are not adjacent. For any $u \in V(G)($ or $u \in G), d_{u}$ represents the degree of $u$. Here we use $N_{u}$ to denote the number of vertices not adjacent to $u \in G$; mathematically,

$$
N_{u}=|\{\omega \mid u \omega \notin E(G)\}|, \quad \text { for each } u \in G .
$$

Forgotten topological index is defined by Gutman and Trinajstic [4].

$$
F(G)=\sum_{v \in V(G)} d_{v}^{3}
$$

Equation (2) can be written as [4]

$$
F(G)=\sum_{u v \in E(G)}\left(d_{u}^{2}+d_{v}^{2}\right)
$$

$\mathrm{Hu}$ et $\mathrm{Al}$. [17] proposed a general form of zeroth-order Randic index given as follows:

$$
{ }^{0} R_{\alpha}(G)=\sum_{u \in G} d_{u}^{\alpha}=\sum_{u v \in E(G)}\left(d_{u}^{\alpha-1}+d_{v}^{\alpha-1}\right)
$$

$\alpha$ is an arbitrary real number.

It can be noted that forgotten index can be obtained for $\alpha=3$. Similarly, Mansour and Song [18] introduced a general formula for zeroth-order Randic coindex as given below:

$$
\overline{{ }^{0} R_{\alpha}}(G)=\sum_{u v \notin E(G)}\left(d_{u}^{\alpha-1}+d_{v}^{\alpha-1}\right) .
$$

For $\alpha=3$, the above formula takes the form of F-coindex used by De et al. [9].

$$
\bar{F}(G)=\sum_{u v \notin E(G)}\left(d_{u}^{2}+d_{v}^{2}\right) .
$$

Very recently, Milovanovi ć et al. [19] proved the following formula for zeroth-order Randic coindex:

$$
\overline{{ }^{0} R_{\alpha}}(G)=\sum_{u \in(G)}\left(n-1-d_{v}\right) d_{v}^{\alpha-1}
$$

for all real values of $\alpha$. Working independantly, Ali et al. [20] published the following forms of F-coindex given by equation (6):

$$
\bar{F}(G)=\sum_{u \in G} d_{u}^{2} \times N_{u}
$$

Also,

$$
\bar{F}(G)=\sum_{u \in G}\left[(n-1) d_{u}^{2}-d_{u}^{3}\right]
$$

where the number of nonadjacent vertices to $u$ is given by

$$
N_{u}=n-1-d_{u} .
$$

The F-coindex is also called Lanzhou Index. In 2021, Dehgardi and Liu [21] characterized trees with fixed maximum degree using the Lanzhou Index. We can easily calculate $F$-coindex, using formula given by equation (8), for some well-known graphs. Let $P_{n}, C_{n}, K_{1, n-1}$, and $K_{n}$ be the path, cycle, star, and complete graphs on $n$ vertices. Then, $F$ coindex for these graphs is as follows:

(1) $\bar{F}\left(P_{n}\right)=4 n^{2}-18 n+20$.

(2) $\bar{F}\left(C_{n}\right)=4 n^{2}-12 n$.

(3) $\bar{F}\left(K_{1, n-1}\right)=(n-1)(n-2)$.

(4) $\bar{F}\left(K_{n}\right)=0$ and $\bar{F}\left(\overline{K_{n}}\right)=0$.

Note here that we reserve $\bar{F}_{u_{i}}$ to represent contribution of $i$ vertices towards $F$-coindex of graph $\bar{F}(G)$.

\section{F-Coindex of Unicyclic Graphs}

Unicyclic graphs are connected graphs with equal number of vertices and edges. Let $U_{n}$ denote the set of the unicyclic graphs with $n$ vertices and $U_{n}^{k}$ denote the class of all unicyclic graphs with $n$ vertices having a cycle of length $k$. Let $U_{n}^{k}\left(p_{1}, p_{2}, \ldots, p_{k}\right) \in U_{n}^{k}$ denote a unicyclic graph with $n$ vertices having cycle of length $k$, and each vertex $i$ of cycle has $p_{i}$ pendant vertices on it, where $1 \leq i \leq k$.

For example, $U_{14}^{3}(5,4,2)$, shown in Figure 1, represents a unicyclic graph with 14 vertices having a cycle of length 3, where these 3 vertices of cycle have 5, 4, and 2 pendants, respectively. It is important to note that in $U_{n}^{k}\left(p_{1}, p_{2}, \ldots, p_{k}\right), k+p_{1}+p_{2}+\ldots+p_{k}=n$.

Theorem 1. Let $G \in U_{n}$ and $d_{u}$ be the degree of vertex $u \in V(G)$. Let $u^{*} \in V(G)$ be the vertex with maximum contribution towards $\bar{F}(G)$; then, degree of the vertex $u^{*}$ is given by

$$
d_{u}^{*}= \begin{cases}\left\lceil\frac{2}{3}(n-1)\right], & \text { if } n \equiv 2(\bmod 3), \\ \left\lfloor\frac{2}{3}(n-1)\right\rfloor, & \text { otherwise. }\end{cases}
$$

Proof. From equation (9), we know that F-coindex of a graph is given by

$$
\bar{F}(G)=\sum_{u \in G}\left[(n-1) d_{u}^{2}-d_{u}^{3}\right]
$$

Let $u^{*}$ with degree $d_{u^{*}}$ have maximum contribution towards $\bar{F}$; then,

$$
\frac{\partial \bar{F}}{\partial d_{u^{*}}}=2(n-1) d_{u^{*}}-3\left(d_{u^{*}}\right)^{2}=0, \quad \text { this gives } d_{u^{*}}=\frac{2(n-1)}{3} .
$$

Also, note that

$$
\frac{\partial^{2} \bar{F}}{\partial d_{u^{*}}^{2}}=-2(n-1)<0
$$

This implies that the vertex with degree $d_{u^{*}}=(2(n-$ $1) / 3$ ) will have maximum contribution towards $\bar{F}(G)$. Note 


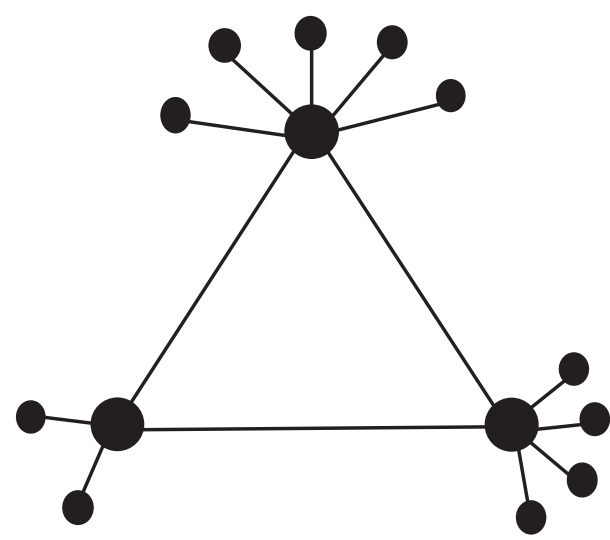

Figure 1: For $U_{16}^{3}(5,4,2)$.

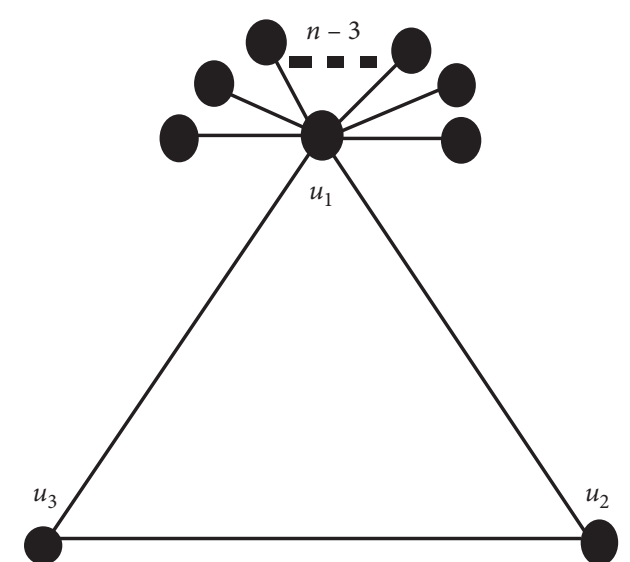

FiguRe 2: For $U_{n}^{3}(n-3,0,0)$.

TABLe 1: For $U_{n}^{3}(n-3,0,0)$.

\begin{tabular}{lcc}
\hline$d_{u}$ & $N_{u}$ & Frequency \\
\hline 1 & $n-2$ & $n-3$ \\
2 & $n-3$ & 2 \\
$n-1$ & 0 & 1 \\
\hline
\end{tabular}

Now we will show that $\bar{F}\left(U_{n}^{3}(n-3,0,0)\right)=(n-3)(n+$ 6 ) is the minimum value of $F$-coindex for the family of unicyclic graph. From equations (8) and (9), contribution towards $F$-coindex of unicyclic graph increases with increase in degree of the vertex until

$$
d_{u^{*}}= \begin{cases}\left\lceil\frac{2}{3}(n-1)\right], & \text { if } n \equiv 2(\bmod 3), \\ \left.\mid \frac{2}{3}(n-1)\right], & \text { otherwise, }\end{cases}
$$

as given in Theorem 1 . We can note from Theorem 1 and equation (9) that the F-coindex can be minimized by minimizing each of the term that is contributing for each of the vertices. This can be further categorized as the following two facts.

Fact\#1 More pendants and two-degree vertices will yield minimum F-coindex.

Fact\#2 Vertex with degree $n-1$ will have zero contribution towards F-coindex of unicyclic graph.

It is also known that total degree of a graph is $2 n$. If one of the vertices has degree $n-1$ with zero contribution towards F-coindex of unicyclic graph, then remaining $n-1$ vertices will have total degree $n+1$. Thus, instead of total degree $2 n$, we are left with the degree $n+1$ that is contributing towards $F$-coindex of unicyclic graph in the form of pendants and two-degree vertices. The only possible structure for such a unicyclic graph is $n-3$ pendants and two vertices of twodegree, that is, $U_{n}^{3}(n-3,0,0)$. It is likewise established that in any other structure of unicyclic graph, there would be more vertices with higher degrees, ultimately contributing more towards $F$-coindex of unicyclic graph than $U_{n}^{3}(n-3,0,0)$.
Thus, $\bar{F}\left(U_{n}^{3}(n-3,0,0)\right)=(n-3)(n+6)$. given in Table 1, we get

$$
\begin{aligned}
\bar{F}\left(U_{n}^{3}(n-3,0,0)\right) & =(n-3)\left[(1)^{2} \cdot(n-2)\right]+2\left[(2)^{2} \cdot(n-3)\right] \\
& =(n-3)(n-2)+8(n-3)=(n-3)(n+6) .
\end{aligned}
$$


Theorem 3. Let $U_{n}^{3}\left(p_{1}, p_{2}, 0\right) \in U_{n}$ with $p_{1}, p_{2}$ be the pendants on $u_{1}$ and $u_{2}$, respectively. If $\left|p_{1}-p_{2}\right| \leq 1$, then the contribution of $u_{1}$ and $u_{2}$ towards $\bar{F}(G)$ is given by

$$
\bar{F}_{u_{i}}=\left\{\begin{array}{ll}
\frac{1}{4}(n+1)^{2}(n-3), & \text { if } n \text { is odd, } \\
\frac{1}{4}\left(n^{3}-n^{2}-6 n-8\right), & \text { if } n \text { is even, }
\end{array} \text { for } i=1,2 .\right.
$$

Also, $\bar{F}_{u_{i}}$, for $i=1,2$, is maximum when $\left|p_{1}-p_{2}\right| \leq 1$.

Proof. Let $U_{n}^{3}\left(p_{1}, p_{2}, 0\right) \in U_{n}$ and $n$ be an odd number and let $p_{1}=p_{2}=((n-3) / 2)$ be the pendants on $u_{1}$ and $u_{2}$, respectively. In this case, both $u_{1}$ and $u_{2}$ will have degree $(1 / 2)(n+1)$ and both will be nonadjacent to $(1 / 2)(n-3)$. Thus, contribution of both of $u_{1}$ and $u_{2}$ towards F-coindex is given by

$$
\begin{array}{r}
\bar{F}_{u_{i}}=2 \cdot\left(\frac{1}{2}(n+1)\right)^{2}\left(\frac{n-3}{2}\right)=\frac{1}{4}(n+1)^{2}(n-3), \\
\text { for } i=1,2 .
\end{array}
$$

Now let us assume that $U_{n}^{3}\left(p_{1}, p_{2}, 0\right) \in U_{n}$ and let $n$ be an even number. Here $u_{1}$ and $u_{2}$ have $p_{1}=(1 / 2)(n-2)$ and $p_{2}=(1 / 2)(n-4), \quad$ respectively. In this case, $N_{u_{1}}=((n-4) / 2)$ and $N_{u_{2}}=((n-2) / 2)$. Thus, we obtain $\bar{F}_{u_{i}}=(1 / 4)\left(n^{3}-n^{2}-6 n-8\right)$, for $i=1,2$.

Now we prove $\bar{F}_{u_{i}}$, for $i=1,2$, is maximum when $\left|p_{1}-p_{2}\right| \leq 1$. On the contrary, assume that, for $\left|p_{1}-p_{2}\right|>1$, contribution of $u^{*}$ and $u^{* *}$ towards $\bar{F}(G)$ is more than $\bar{F}_{u_{i}}$ for $i=1,2$. This means that there exists a positive integer $m \geq 1$ such that pendant at vertex $u^{*}$ is $p_{1}=((n-3) / 2)+m$ and similarly for $u^{* *}, p_{2}=((n-3) / 2)-m$ and

$$
\bar{F}_{u^{*}, u^{* *}}>\bar{F}_{u_{i}}, \quad \text { for } i=1,2 .
$$

Initially, we assume that $n$ is odd. It is easy to see that

$$
\begin{aligned}
d_{u^{*}} & =\frac{n+1}{2}+m, \\
d_{u^{* *}} & =\frac{n+1}{2}-m .
\end{aligned}
$$

Note that

$$
\begin{gathered}
N_{u^{*}}=\frac{n+1}{2}-m, \\
N_{\mathcal{U}^{* *}}=\frac{n+1}{2}+m .
\end{gathered}
$$

Thus,

$$
\begin{aligned}
\bar{F}_{u^{*}, u^{* *}} & =\left(\frac{n+1}{2}+m\right)^{2}\left(\frac{n+1}{2}-m\right)+\left(\frac{n+1}{2}-m\right)^{2}\left(\frac{n+1}{2}+m\right) \\
& =\frac{1}{4}(n+1)^{2}(n-3)-\left[n m^{2}+n^{2} m+n m+5 m^{2}\right] \\
& <\frac{1}{4}(n+1)^{2}(n-3), \quad \text { since } m, n \geq 1 .
\end{aligned}
$$

Now assume that $n$ is even; then, we have

$$
\begin{aligned}
d_{u^{*}} & =\frac{n+2}{2}+m, \\
d_{u^{* *}} & =\frac{n}{2}-m .
\end{aligned}
$$

Note that

$$
\begin{aligned}
N_{u^{*}} & =\frac{n-4}{2}-m, \\
N_{u^{* *}} & =\frac{n-2}{2}+m,
\end{aligned}
$$

which gives

$$
\begin{aligned}
\bar{F}_{u^{*}, \mathcal{u}^{* *}}= & \left(\frac{n+2}{2}+m\right)^{2}\left(\frac{n-4}{2}-m\right)+\left(\frac{n}{2}-m\right)^{2}\left(\frac{n+2}{2}+m\right) \\
= & \frac{1}{4}\left(n^{3}-n^{2}-6 n-8\right)-[m(m+1)(n+5)] \\
& <\frac{1}{4}\left(n^{3}-n^{2}-6 n-8\right), \quad \text { since } m, n \geq 1 .
\end{aligned}
$$

It is obvious that our assumption that equation (21) holds is wrong. Therefore, $\bar{F}_{u_{i}}$, for $i=1,2$, is maximum when $\left|p_{1}-p_{2}\right| \leq 1$.

It is important to note here that if one vertex has degree $d_{u^{*}}$ given by (11), then maximum possible degree of any 
other vertex, say $u^{* *}$, in a unicyclic graph would be $n-3-d_{u^{*}}$. Theorem 3 says that the contribution of both $u^{*}$ and $u^{* *}$ towards $\bar{F}(G)$ would be less than that if both vertices having degrees states in Theorem 3.

Theorem 4. Let $U_{n}^{k}\left(p_{1}, p_{2}, \ldots, p_{k}\right) \in U_{n}^{k}$ such that $\left|p_{i}-p_{j}\right| \leq 1$, for $1 \leq i, j \leq k$; then, $k=(2 n /(n-4))$ maximizes F-coindex of unicyclic graph.
Proof. Without loss of generality, we assume $n$ such that each vertex has equal vertices, that is, $(1 / k)(n-k)$. From Figure 3, we can have the following table.

It is obvious from the structure of $U_{n}^{k}\left(p_{1}, p_{2}, \ldots, p_{k}\right)$ that there would be $(n-k)$ vertices of degree 1 and $k$ vertices of degree $(1 / k)(n+k)$ as shown in Figure 3.

Using the formula given by equation (8) and data given in Table 2, we get

$$
\begin{aligned}
\bar{F}\left(U_{n}^{k}\left(p_{1}, p_{2}, \ldots, p_{k}\right)\right)= & k\left[\frac{n-k}{k}+2\right]^{2}\left[\frac{n(k-1)-2 k}{k}\right]+\left[(1)^{2}(n-k)(n-2)\right] \\
= & \frac{1}{k^{2}}\left[k^{3}(n-2)+k^{2}\left(2 n^{2}-5 n\right)+k\left(n^{3}-4 n^{2}\right)-n^{3}\right] \\
& +n^{2}-k n-2 n+2 k \\
= & \frac{1}{k}\left(n^{3}-4 n^{2}\right)-\frac{n^{3}}{k^{2}}+\left(3 n^{2}-7 n\right) .
\end{aligned}
$$

In order to get the value of that maximizes $\bar{F}\left(U_{n}^{k}\left(p_{1}, p_{2}, \ldots, p_{k}\right)\right)$, we proceed as follows.

$$
\frac{\mathrm{d} \bar{F}}{\mathrm{~d} k}=0, \quad \text { implies that } k=\frac{2 n}{n-4},
$$

Also, note that for $k=(2 n /(n-4))$, we have $\left(\mathrm{d}^{2} \bar{F} / \mathrm{d} k^{2}\right)<0$. This means that $k=(2 n /(n-4))$ maximizes $\bar{F}\left(U_{n}^{k}\left(p_{1}, p_{2}, \ldots, p_{k}\right)\right)$.

We know that $k$ must be a positive integer. From the expression $k=(2 n /(n-4))$, it can be seen that for large values of $n, k$ approaches 2 but for values of $n$ close to $11, k$ is closer to 3 .

Theorem 5. For $n \geq 28$, let $U_{n}^{3}\left(p_{1}, p_{2}, 0\right) \in U_{n}^{k}$, with $p_{1}, p_{2} \in\{\lfloor(n-3) / 2\rfloor,\lceil(n-3) / 2\rceil\}$, such that $p_{1}+p_{2}=n-3$. In this case,

$$
\bar{F}\left(U_{n}^{3}\left(p_{1}, p_{2}, 0\right)\right)= \begin{cases}\frac{1}{4}(n-3)\left(n^{2}+6 n+9\right), & \text { if } n \text { is odd, } \\ \frac{1}{4}\left(n^{3}+3 n^{2}-10 n-32\right), & \text { if } n \text { is even. }\end{cases}
$$

Furthermore, values given by equation (30) are maximum $F$-coindex for the family of unicyclic graphs with $n \geq 28$.

Proof. Let $U_{n}^{3}\left(p_{1}, p_{2}, 0\right) \in U_{n}^{k}$, with $p_{1}, p_{2} \in \mathbb{N}$ such that $p_{1}+p_{2}=n-3$ and

$$
\left|p_{1}-p_{2}\right|=\left\{\begin{array}{ll}
1, & \text { if } n \text { is even, } \\
0, & \text { if } n \text { is odd, }
\end{array} \quad \text { for } n \geq 28\right.
$$

The structure of such graphs is shown in Figure 4 .
From Figure 4, Tables 3 and 4 are tabulated. In this case, F-coindex is obtained by adding to $(n-3)(n+2)$ the contribution of pendants and degree 2 vertices in equation (19), respectively for odd and even values of $n$. Thus using the table given above, we may write

$$
\bar{F}\left(U_{n}^{3}\left(p_{1}, p_{2}, 0\right)\right)= \begin{cases}\frac{1}{4}(n-3)\left(n^{2}+6 n+9\right), & \text { if } n \text { is odd, } \\ \frac{1}{4}\left(n^{3}+3 n^{2}-10 n-32\right), & \text { if } n \text { is even. }\end{cases}
$$

It can easily be verified that $\bar{F}\left(U_{n}^{3}\left(p_{1}, p_{2}, 0\right)\right)$ given by equation (32) is maximum by using Theorems 3 and 4 .

Theorem 6. For $11 \leq n \leq 27$, let $U_{n}^{3}\left(p_{1}, p_{2}, p_{3}\right) \in U_{n}^{k}$ with $p_{i} \in\{\lfloor(n-3) / 3\rfloor,\lceil(n-3) / 3\rceil\}$, for $i=1,2,3$, such that $\sum_{i=1}^{3} p_{i}=n-3 ;$ then,

$$
\bar{F}\left(U_{n}^{3}\left(p_{1}, p_{2}, p_{3}\right)\right)= \begin{cases}\frac{2 n^{3}}{9}+\frac{5 n^{2}}{3}-7 n, & \text { if } n \equiv 0(\bmod 3), \\ \frac{6 n^{3}}{27}+\frac{5 n^{2}}{3}-\frac{53 n}{9}-4, & \text { if } n \equiv 1(\bmod 3), \\ \frac{2 n^{3}}{9}+\frac{53 n^{2}}{27}-7 n-\frac{22}{9}, & \text { if } n \equiv 2(\bmod 3) .\end{cases}
$$

Proof. For $11 \leq n \leq 27$, let $U_{n}^{3}\left(p_{1}, p_{2}, p_{3}\right) \in U_{n}^{k}$ with $p_{1}, p_{2}, p_{3} \in \mathbb{N}$ such that $p_{1}+p_{2}+p_{3}=n-3$; then, we have following three possible cases for $p_{1}, p_{2}$, and $p_{3}$.

Case I: when $n \equiv 0(\bmod 3)$, then $p_{1}=p_{2}=p_{3}=$ $((n-3) / 3)$. 
TABLE 2: For $U_{n}^{k}\left(p_{1}, p_{2}, \ldots, p_{k}\right)$ with $p_{i}=(1 / k)(n-k)$, for $1 \leq i \leq k$.

\begin{tabular}{lcc}
\hline$d_{u}$ & $N_{u}$ & Frequency \\
\hline 1 & $n-2$ & $n-k$ \\
$((n-k) / k)+2$ & $((n(k-1)-2 k) / k)$ & $k$ \\
\hline
\end{tabular}

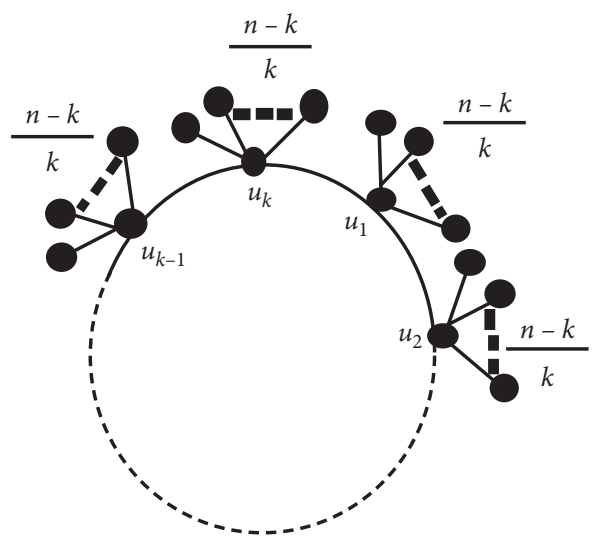

FIGURE 3: For $U_{n}^{k}\left(p_{1}, p_{2}, \ldots, p_{k}\right)$ with $p_{i}=(1 / k)(n-k)$, for $1 \leq i \leq k$.

Case II: when $n \equiv 1(\bmod 3)$, then $p_{1}=\lceil(n-3) / 3\rceil$ and $p_{2}=p_{3}=\lfloor(n-3) / 3\rfloor$.

Case III: when $n \equiv 2(\bmod 3)$, then $p_{1}=p_{2}=\lceil(n-3) /$ $3\rceil$ and $p_{3}=\lfloor(n-3) / 3\rfloor$.

Now we consider each case separately, to find the F-coindex of $U_{n}^{3}\left(p_{1}, p_{2}, p_{3}\right)$ for $11 \leq n \leq 27$.

Case I: when $n \equiv 0(\bmod 3)$, then $p_{1}=p_{2}=p_{3}=$ $((n-3) / 3)$, as shown in Figure 5(a). In this class, we discuss all graphs for $11 \leq n \leq 27$ when $n$ is divisible by 3 , that is, $n=3 k$, for $k=4,5, \ldots, 9$. For such graphs, degree of each vertex of cycle is given by $((n+3) / 3)$ and there are $(n-3)$ pendant vertices. From the figure, we have the following table.

Using the formula given by equation (8) and Table 5, we get

$$
\bar{F}(G)=3 \cdot\left(\frac{n+3}{3}\right)^{2}\left(\frac{2}{3}(n-3)\right)+(n-2)(n-3) .
$$

Simplification of the above equation gives

$$
\bar{F}(G)=\frac{2 n^{3}}{9}+\frac{5 n^{2}}{3}-7 n .
$$

Case II: when $n \equiv 1(\bmod 3)$, then $p_{1}=((n-1) / 3)$ and $p_{2}=p_{3}=((n-4) / 3)$, for $n=3 k+1$ where $k=4,5, \ldots, 8$, as shown in Figure $5(\mathrm{~b})$.

In this case, we have the following values of degrees, nonadjacent vertices, and frequencies. Using the formula given by equation (8) and Table 6, we get

$$
\begin{aligned}
\bar{F}(G)= & 2\left(\frac{n+2}{3}\right)^{2}\left(\frac{2 n-5}{3}\right)+\left(\frac{n+5}{3}\right)^{2}\left(\frac{2 n-8}{3}\right) \\
& +(n-3)(n-2) .
\end{aligned}
$$

After simplifying last equation, we obtain

$$
\bar{F}(G)=\frac{6 n^{3}}{27}+\frac{5 n^{2}}{3}-\frac{53 n}{9}-4
$$

Case III: in the last case, we consider the family of unicyclic graphs $U_{n}^{3}\left(p_{1}, p_{2}, p_{3}\right) \in U_{n}^{k}$ with $n \equiv 2((\bmod 3))$. Here $p_{1}=p_{2}=((n-2) / 3)$ and $p_{3}=((n-5) / 3)$ where $n=3 k+2$ for $k=$ $4,5, \ldots, 8$ (Figure $5(\mathrm{c})$ ). It is easy to construct the following table.

Using the formula given by equation (8) and Table 7, we get

$$
\begin{aligned}
\bar{F}(G)= & 2\left(\frac{n+4}{3}\right)^{2}\left(\frac{2 n-7}{3}\right)+\left(\frac{n+1}{3}\right)^{2}\left(\frac{2 n-4}{3}\right) \\
& +(n-3)(n-2) .
\end{aligned}
$$

Thus, we obtain

$$
\bar{F}(G)=\frac{2 n^{3}}{9}+\frac{53 n^{2}}{27}-7 n-\frac{22}{9} .
$$

Theorem 7. Let $U_{n}^{3}\left(p_{1}, p_{2}, p_{3}\right) \in U_{n}^{k}$ with $p_{i} \in\{\lfloor(n$ $-3) / 3\rfloor,\lceil(n-3) / 3\rceil\}$, for $i=1,2,3$, such that $p_{1}+p_{2}+$ $p_{3}=n-3$; then, $\bar{F}\left(U_{n}^{3}\left(p_{1}, p_{2}, p_{3}\right)\right)$ has maximum F-coindex for the family of unicyclic graphs with $11 \leq n \leq 27$.

Proof. By Theorem 4, we know that $k=(2 n /(n-4))$ maximizes F-coindex of unicyclic graph. This value must be integer as it represents number of vertices on which we should divide pendant vertices. The value of $k$ given in Theorem 4 is close to 3 when $n=11$ and it decreases as we increase $n$. Therefore, we can compare $F$-coindex of both values of $k$, that is, $k=2$ and $k=3$.

Without loss of generality, we assume that $n-3$ is even and divisible by 3 , and it is equivalent to saying that $n$ is odd and divisible by 3 . It is evident from Figure 6 and Tables 8 and 9 that both $U_{n}^{3}\left(p_{1}, p_{2}, p_{3}\right)$ and $U_{n}^{3}\left(p_{1}, p_{2}, 0\right)$ have same pendant vertices with same number of nonadjacent vertices.

Therefore, contribution of pendant vertices would be same in both cases. It means that we should compare the contribution of other vertices for $F$-coindex of $U_{n}^{3}\left(p_{1}, p_{2}, p_{3}\right)$ and $U_{n}^{3}\left(p_{1}, p_{2}, 0\right)$. We can see that in $U_{n}^{3}\left(p_{1}, p_{2}, p_{3}\right)$, there are 3 vertices, say $u_{1}, u_{2}$, and $u_{3}$, with degree $((n+3) / 3)$ with nonadjacent vertices $((2(n-3)) / 3)$.

$$
\bar{F}_{u_{i}}=\frac{2}{9}(n+3)^{2}(n-3), \quad \text { for } i=1,2,3,
$$




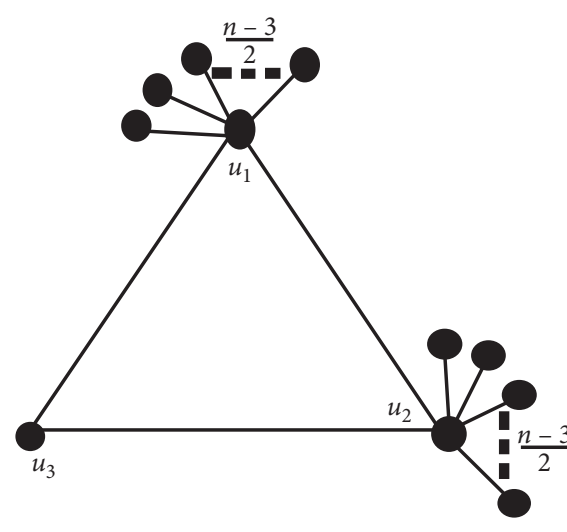

(a)

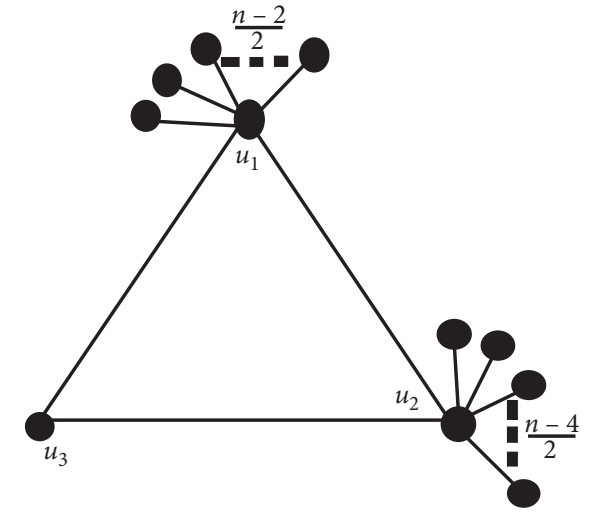

(b)

FIgURE 4: $U_{n}^{3}\left(p_{1}, p_{2}, 0\right), n \geq 28$. (a) $n$ is odd. (b) $n$ is even.

TABLE 3: For $U_{n}^{3}(((n-3) / 2),((n-3) / 2), 0)$.

\begin{tabular}{lcc}
\hline$d_{u}$ & $N_{u}$ & Frequency \\
\hline 1 & $n-2$ & $n-3$ \\
2 & $n-3$ & 1 \\
$(1 / 2)(n+1)$ & $(1 / 2)(n-3)$ & 2 \\
\hline
\end{tabular}

TABLe 4: For $U_{n}^{3}(((n-2) / 2),((n-4) / 2), 0)$.

\begin{tabular}{lcc}
\hline$d_{u}$ & $N_{u}$ & Frequency \\
\hline 1 & $n-2$ & $n-3$ \\
2 & $n-3$ & 1 \\
$(n / 2)$ & $(1 / 2)(n-2)$ & 1 \\
$(1 / 2)(n+2)$ & $(1 / 2)(n-4)$ & 1 \\
\hline
\end{tabular}

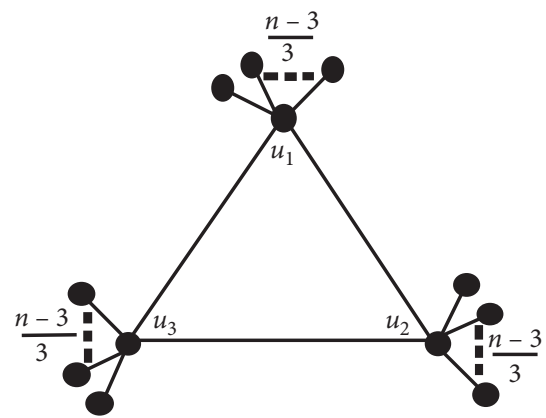

(a)

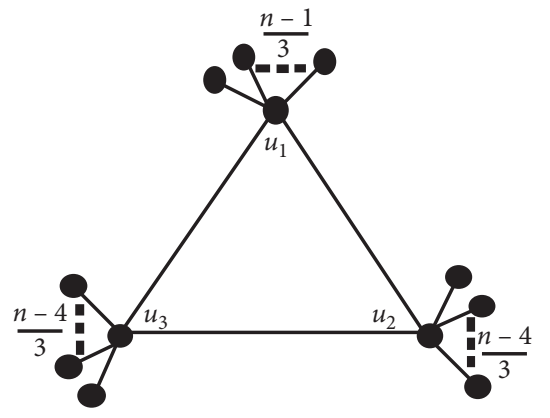

(b)

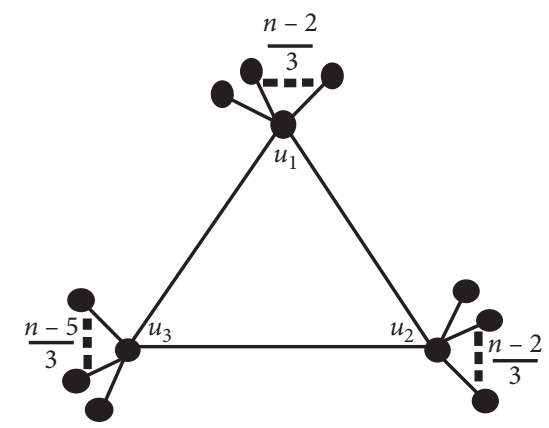

(c)

FIgURE 4: $U_{n}^{3}\left(p_{1}, p_{2}, p_{3}\right)$ with $p_{i} \in\{\lfloor(n-3) / 3\rfloor,\lceil(n-3) / 3\rceil\}$, for $i=1,2,3$.

TABLE 5: For $U_{n}^{3}\left(p_{1}, p_{2}, p_{3}\right)$.

\begin{tabular}{lcc}
\hline$d_{u}$ & $N_{u}$ & Frequency \\
\hline$((n+3) / 3)$ & $(2 / 3)(n-3)$ & 3 \\
1 & $n-2$ & $n-3$ \\
\hline
\end{tabular}

TABle 6: For $U_{n}^{3}\left(p_{1}, p_{2}, p_{3}\right)$.

\begin{tabular}{lcc}
\hline$d_{u}$ & $N_{u}$ & Frequency \\
\hline$(1 / 3)(n+2)$ & $(1 / 3)(2 n-5)$ & 2 \\
$(1 / 3)(n+5)$ & $(2 / 3)(n-4)$ & 1 \\
1 & $n-2$ & $n-3$ \\
\hline
\end{tabular}


TABLE 7: For $U_{n}^{3}\left(p_{1}, p_{2}, p_{3}\right)$.

\begin{tabular}{lcc}
\hline$d_{u}$ & $N_{u}$ & Frequency \\
\hline$(1 / 3)(n+4)$ & $(1 / 3)(2 n-7)$ & 2 \\
$(1 / 3)(n+1)$ & $(2 / 3)(n-2)$ & 1 \\
1 & $n-2$ & $n-3$ \\
\hline
\end{tabular}

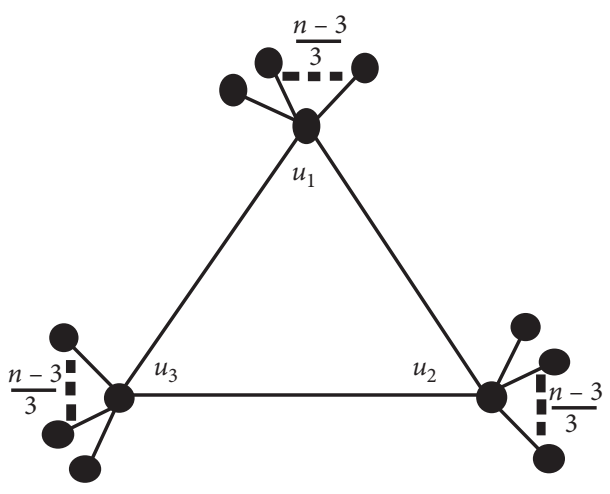

(a)

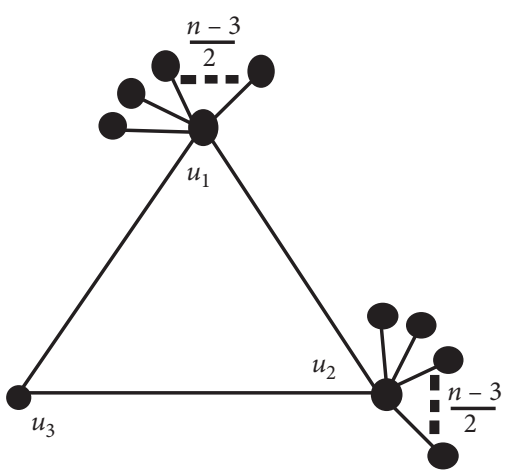

(b)

FIgURE 4: $U_{n}^{3}\left(p_{1}, p_{2}, p_{3}\right)$ and $U_{n}^{3}\left(p_{1}, p_{2}, 0\right)$.

TABLE 8: For $U_{n}^{3}(((n-3) / 3), t((n-3) / 3) n, q((n-3) / 3))$.

\begin{tabular}{lcc}
\hline$d_{u}$ & $N_{u}$ & Frequency \\
\hline$((n+3) / 3)$ & $(2 / 3)(n-3)$ & 3 \\
1 & $n-2$ & $n-3$ \\
\hline
\end{tabular}

TABLE 9: For $U_{n}^{3}(((n-3) / 2),((n-3) / 2), 0)$.

\begin{tabular}{lcc}
\hline$d_{u}$ & $N_{u}$ & Frequency \\
\hline 1 & $n-2$ & $n-3$ \\
2 & $n-3$ & 1 \\
$((n+1) / 2)$ & $((n-3) / 2)$ & 2 \\
\hline
\end{tabular}

where $\bar{F}_{u_{i}}$, for $i=1,2,3$, represents contribution of $u_{1}, u_{2}$, and $u_{3}$ towards $\bar{F}\left(U_{n}^{3}(((n-3) / 3),((n-3) / 3),((n-3) / 3))\right)$.

Similarly, for $U_{n}^{3}\left(p_{1}, p_{2}, 0\right)$, contribution of $v_{1}, v_{2}$, and $v_{3}$ is given by

$$
\bar{F}_{v_{i}}=\frac{1}{4}(n-3)\left(n^{2}+2 n+17\right), \quad \text { for } \quad i=1,2,3,
$$

where $\bar{F}_{v_{i}}$, for $i=1,2,3$, represents contribution of $v_{1}, v_{2}$, and $v_{3}$ towards $\bar{F}\left(U_{n}^{3}(((n-3) / 2),((n-3) / 2)), 0\right)$. By analyzing equations (40) and (41), we have

$\bar{F}_{u_{i}}=\bar{F}_{v_{i}}, \quad$ for $n=27$, and $\bar{F}_{u_{i}}>\bar{F}_{v_{i}}$ for $11 \leq n<27$.

In general, we can write

$\begin{cases}\bar{F}\left(U_{n}^{3}\left(p_{1}, p_{2}, p_{3}\right)\right)>\bar{F}\left(U_{n}^{3}\left(p_{1}, p_{2}, 0\right)\right), & \text { for } 11 \leq n<27, \\ \bar{F}\left(U_{n}^{3}\left(p_{1}, p_{2}, p_{3}\right)\right)=\bar{F}\left(U_{n}^{3}\left(p_{1}, p_{2}, 0\right)\right), & \text { for } n=27, \\ \bar{F}\left(U_{n}^{3}\left(p_{1}, p_{2}, p_{3}\right)\right)<\bar{F}\left(U_{n}^{3}\left(p_{1}, p_{2}, 0\right)\right), & \text { for } n>27 .\end{cases}$
Therefore, for $11 \leq n \leq 27, \bar{F}\left(U_{n}^{3}\left(p_{1}, p_{2}, p_{3}\right)\right)$ has maximum $F$-coindex for the family of unicyclic graphs.

\section{Conclusions}

In this paper, we introduced an alternative form of $F$ coindex of graphs. We used this form to study different properties of unicyclic graphs. We also calculated maximum and minimum F-coindex for the family of unicyclic graphs.

This alternative form may be used to study F-coindex of some special and chemically interesting graphs. It may help in studying $F$-coindex of other classes of graphs like bicyclic and tricyclic classes. Furthermore, it would be an important direction if one can explore similar formula for other topological coindices.

\section{Data Availability}

The data used to support the findings of this study are included within the article.

\section{Conflicts of Interest}

The authors declare that they have no conflicts of interest.

\section{References}

[1] M. Imran, M. Siddiqui, M. Naeem, and M. Iqbal, "On topological properties of symmetric chemical structures," Symmetry, vol. 10, no. 5, 173 pages, 2018.

[2] S. Kang, Z. Iqbal, M. Ishaq, R. Sarfraz, A. Aslam, and W. Nazeer, "On eccentricity-based topological indices and polynomials of phosphorus-containing dendrimers," Symmetry, vol. 10, no. 7, 237 pages, 2018. 
[3] G. Rcker and C. Rcker, "On topological indices, boiling points, and cycloalkanes," Journal of Chemical Information and Modeling, vol. 39, pp. 788-802, 1999.

[4] I. Gutman and N. Trinajstić, "Graph theory and molecular orbitals. Total $\varphi$-electron energy of alternant hydrocarbons," Chemical Physics Letters, vol. 17, no. 4, pp. 535-538, 1972.

[5] S. Nikolic', G. Kovacevic', A. Milicevic', and N. Trinajstic', "The Zagreb indices 30 Years after," Croatica Chemica Acta, vol. 76, pp. 113-124, 2003, ISSN-0011-1643.

[6] B. Furtula and I. Gutman, "A forgotten topological index," Journal of Mathematical Chemistry, vol. 53, no. 4, pp. 11841190, 2015.

[7] T. Doslic, "Vertex-weighted Wiener polynomials for composite graphs," Ars Mathematica Contemporanea, vol. 1, pp. 66-80, 2008.

[8] A. R. Ashrafi, T. Došlić, and A. Hamzeh, "The Zagreb coindices of graph operations," Discrete Applied Mathematics, vol. 158, no. 15, pp. 1571-1578, 2010.

[9] N. De, S. M. A. Nayeem, and A. Pal, "The F-coindex of some graph operations," Springerplus, vol. 5, no. 1, 5 pages, 2016.

[10] H. Abdo, D. Dimitrov, and I. Gutman, "On extremal trees with respect to the F-index," Kuwait Journal of Science, vol. 44, pp. 1-8, 2017.

[11] Z. Che and Z. Chen, "Lower and upper bounds of the forgotten topological index," MATCH Communications in Mathematical and in Computer Chemistry, vol. 76, pp. 635648, 2016.

[12] N. De, "F-index and coindex of some derived graphs," Bulletin of International Mathematical Virtual Institute, vol. 8, pp. 81-88, 2018.

[13] W. Gao, M. R. Farahani, and L. Shi, "Forgotten topological index of some drug structures," Acta Medica Mediterranea, vol. 32, pp. 579-585, 2016.

[14] B. Yasir, A. Adnan, K. Muhammad et al., "On forgotten topological indices of some dendrimers structure," Molecules, vol. 22867 pages, 2017.

[15] R. Amin and S. M. A. Nayeem, "Trees with minimum Fcoindex," Electronic Notes in Discrete Mathematics, vol. 63, pp. 303-310, 2017.

[16] S. Akhter, M. Imran, and M. R. Farahani, "Extremal unicyclic and bicyclic graphs with respect to the F-index," AKCE International Journal of Graphs and Combinatorics, vol. 14, no. 1, pp. 80-91, 2017.

[17] Y. Hu, X. Li, T. Xu, and I. Gutman, "On molecular graphs with smallest and greatest zeroth?order Randic index," MATCH Communications in Mathematical and in Computer Chemistry, vol. 54, pp. 425-434, 2005.

[18] T. Mansour and C. Song, "The and -analogs of Zagreb indices and coindices of graphs," International Journal of Combinatorics, vol. 2012, pp. 1-10, 2012.

[19] I. Milovanovi, M. Matejic, and E. Milovanovi, "A note on the general zeroth-order Randic coindex of graphs," Contributions to Mathematics, vol. 1, pp. 17-21, 2020.

[20] Y. Ali, Z. Bibi, and Q. Kiran, "Forgotten coindex of some nontoxic dendrimers structure used in targeted drug delivery," Main Group Metal Chemistry, vol. 44, no. 1, pp. 22-31, 2021.

[21] N. Dehgardi and J. Liu, "Lanzhou index of trees with fixed maximum degree," MATCH Communications in Mathematical and in Computer Chemistry, vol. 86, pp. 3-10, 2021. 